\title{
Hamiltonian, Lagrangian and Physical Laws
}

\author{
E. Comay \\ Charactell Ltd., Tel-Aviv, Israel \\ Email: elicomay@post.tau.ac.il
}

How to cite this paper: Comay, E. (2019) Hamiltonian, Lagrangian and Physical Laws. Open Access Library Journal, 6: e5536. https://doi.org/10.4236/oalib.1105536

Received: June 12, 2019

Accepted: July 15, 2019

Published: July 18, 2019

Copyright $\odot 2019$ by author(s) and Open Access Library Inc.

This work is licensed under the Creative Commons Attribution International License (CC BY 4.0).

http://creativecommons.org/licenses/by/4.0/

\begin{abstract}
The significance of the correspondence between the classical limit of quantum theories and the laws of classical physics is explained. It is proved that this correspondence yields constraints on acceptable quantum theories. The variational principle is taken as the basis of the analysis. The discussion shows that the first order Dirac equation abides by these constraints, whereas second order quantum equations fail to do that.
\end{abstract}

\section{Subject Areas}

Theoretical Physics

\section{Keywords}

The Correspondence Principle, Constraints on Quantum Theories, the Variational Principle, First Order Quantum Equations, Second Order Quantum Equations

\section{Introduction}

The correspondence between quantum theories and classical physics is an important element of the analysis presented below. Considering a closed system of $n$ classical particles, the principle of least action can be used for a derivation of the system's equations of motion. These equations are derived from a Lagrangian of the form

$$
L(q, \dot{q}, t) .
$$

Here $q$ denotes $3 n$ generalized coordinates that are the particles' location, $\dot{q}$ denotes the time derivatives of these coordinates, and $t$ denotes the time (see [1], p. 2).

The principle of least action yields for every generalized coordinate $q_{i}$ an Euler-Lagrange equation 


$$
\frac{\mathrm{d}}{\mathrm{d} t}\left(\frac{\partial L}{\partial \dot{q}_{i}}\right)-\frac{\partial L}{\partial q_{i}}=0
$$

This is a set of $3 n$ second order differential equations, and a specific solution is obtained for every set of $6 n$ appropriate initial conditions which depend on $q, \dot{q}$.

It is well known that this Lagrangian formalism is not a unique theoretical description of the system. The mathematical procedure called Legendre transformation casts the Lagrangian formalism to an expression which depends on a different set of independent variables. An important example is the Hamiltonian $H(p, q, t)$ which depends on $n$ generalized coordinates and on $n$ conjugate momenta $p$. Thus, one derives a Hamiltonian from a given Lagrangian

$$
H(p, q, t)=\sum_{i} p_{i} \dot{q}_{i}-L(q, \dot{q}, t)
$$

(see [1], p. 131, [2], p. 337). The Hamilton equations of motion are

$$
\dot{q}_{i}=\partial H / \partial p_{i} ; \dot{p}_{i}=-\partial H / \partial q_{i}
$$

(see [1], p. 132, [2], p. 337).

The number of degrees of freedom of the initial conditions of the Lagrangian formalism (1), (2) is the same as that of the Hamiltonian formalism (3), (4). This evidence indicates the mathematical equivalence of these formalisms (see [1], p. 131, [2], p. 334). However, it is interesting to point out that unlike the Lagrangian formalism that yields differential equations of the second order, the equations of motion of the Hamiltonian formalism (4) are first order differential equations.

An important property of an elementary classical particle is its pointlike form (see [3], pp. 46-47). Therefore, the general description of a system of elementary classical particles takes the following structure. A set of coordinates is defined. (This set is sometimes called background.) The position and the velocity of each particle are described by appropriate products of Dirac delta functions of the coordinates. The equations of motion yield a specific solution for every appropriate set of initial conditions.

The main objective of the present work is to examine the Lagrangian formalism and the Hamiltonian formalism in the quantum domain. The classical limit of quantum mechanics is an element of the analysis. The success of classical mechanics means that "classical mechanics must therefore be a limiting case of quantum mechanics" ([4], p. 84, see also [5], pp. 25-27, 137, 138; [6]). Furthermore, quantum field theory (QFT) corresponds to quantum mechanics. For example, a well-known textbook states: "First, some good news: quantum field theory is based on the same quantum mechanics that was invented by Schroedinger, Heisenberg, Pauli, Born, and others in 1925-26, and has been used ever since in atomic, molecular, nuclear and condensed matter physics" (see [7], p. 49). This statement means that there are certain relationships between quantum theories and classical physics. Below, these relationships are called the 
Weinberg correspondence principle. A general discussion of the correspondence between physical theories is presented on pp. 3 - 6 of [8].

It turns out that quantum theories cannot directly use a classical Lagrangian or a classical Hamiltonian as a basis for the theory. This problem stems from the uncertainty relations

$$
\Delta x \cdot \Delta p_{x} \geq \hbar .
$$

Indeed, if $q_{i}, \dot{q}_{i}$ are well-defined quantities then the conjugate momentum $p_{i}=\partial L / \partial \dot{q}_{i}$ is well defined, and the uncertainty relation (5) is violated. Hence, a Lagrangian of the form (1) cannot be used in quantum theories.

The same is true with the Hamiltonian formalism of classical physics (3). Here the coordinates and their conjugate momenta are used as independent variables. However, the uncertainty relations (5) proves that a coordinate and its conjugate momentum cannot be simultaneously used as independent variables.

The previous arguments explain why the mathematical structure of quantum theories differs from that of classical physics. Therefore, a quantum theory should be constructed on the basis of different principles. However, the correspondence between classical physics and the classical limit of quantum theories is a constraint on this limit. The main objective of the present work is to examine the Lagrangian and the Hamiltonian of quantum theories. The discussion is restricted to quantum theories of elementary massive particles.

Units where $\hbar=c=1$ are used. Greek indices run from 0 to 3 . Most formulas take the standard form of a relativistic covariant expression. The metric is diagonal and its entries are $(1,-1,-1,-1)$. The second section shows constraints on quantum theories. The third section shows that the first order Dirac equation is compatible with these constraints. By contrast, the fourth section proves that second order quantum functions are incompatible with these constraints. The analysis shown in the fifth section approves the results of the fourth section. The last section summarizes this work.

\section{Constraints on a Quantum Theory}

Constraints that are relevant to quantum theories are presented below. Classical physics is consistent with several conservation laws, like that of energy, momentum, angular momentum and charge. Therefore, the classical limit of a quantum theory should abide by these laws. The general approach to this issue is to write an appropriate Lagrangian density that yields the quantum equation, and to use the Noether theorem which connects symmetries of this Lagrangian density with conservation laws that are satisfied by the quantum differential equation (see [9], pp. 17-22).

The de Broglie principle says that a quantum particle has wave properties. Hence, an elementary quantum particle is described by a wave function $\psi(x)$ which depends on the four space-time coordinates $x=(t, x)$. This form of $\psi(x)$ indicates that an elementary quantum particle is pointlike. Indeed, the four space-time coordinates $x$ can describe the probability of the particle's 
location at $x$, but these variables cannot determine the degrees of freedom that are required for a description of the internal structure of a composite particle.

These arguments show that a quantum theory of an elementary particle depends on a Lagrangian density of the following form

$$
\mathcal{L}\left(\psi(x), \psi(x)_{, \mu}\right) .
$$

Special relativity requires that the Lagrangian density (6) is a Lorentz scalar. The Euler-Lagrange equation of (6) is

$$
\frac{\partial \mathcal{L}}{\partial \psi}-\frac{\partial}{\partial x^{\mu}} \frac{\partial \mathcal{L}}{\partial\left(\partial \psi / \partial x^{\mu}\right)}=0
$$

(see [7], p. 300). In the case of several functions, one derives an appropriate equation for each function. These equations are regarded as the equations of motion of the quantum system. As a matter of fact, this approach is adopted by the present mainstream textbooks. For example: "All field theories used in current theories of elementary particles have Lagrangians of this form" (see [7], p. 300). This kind of Lagrangian scheme is used below.

Another requirement imposed on a physical theory is an adequate description of experimental results that are carried out within the theory's domain of validity. A measurement device whose state changes with time is an important element of an experiment. Hence, the required quantum equation must take the form of a time-dependent differential equation. Time is the 0 -component of the 4 -vector $(t, \boldsymbol{x})$.

Moreover, an essential aspect of measurement is a change of the system's state. Therefore, the Lagrangian density of a quantum particle must contain an interaction term that produces the transition between the initial state and the final state of the quantum system.

Important cases of time-dependent quantum transitions are described by the Fermi Golden Rule (see [10], pp. 475-481). The transition probability per unit time is proportional to the square of the Hamiltonian's matrix element

$$
w \propto|\langle f|V| i\rangle|^{2} .
$$

Here $|i\rangle$ denotes the initial state, $\langle f|$ denotes the final state, and $V$ is the interaction part of the Hamiltonian. The Hamiltonian is an energy operator, and for this reason it is a 0 -component of a 4 -vector. This property indicates its relationships to the above mentioned time-dependent processes.

The Fermi Golden Rule (8) is an illustration of the significance of the Hamiltonian in quantum mechanics. The Weinberg correspondence principle means that the Hamiltonian is also an important element of QFT. QFT theories depend on the Lagrangian density (6). Therefore, the first step aims to obtain an expression for the corresponding Hamiltonian density. A particle's density is the 0-component of a 4-vector (see [3], pp. 73-75), and energy is the 0-component of the energy-momentum 4-vector. Hence, the Hamiltonian density is the 00 -entry of a second rank tensor, called the energy-momentum tensor. This 
tensor is derived from the Lagrangian density $\mathcal{L}$

$$
T^{\mu v}=\sum_{n} \frac{\partial \mathcal{L}}{\partial \psi_{, v}^{(n)}} g^{\mu \lambda} \psi_{, \lambda}^{(n)}-g^{\mu v} \mathcal{L},
$$

where $n$ runs on all independent fields (see [3], p. 83; [11], p. 310). The $T^{00}$ entry of this tensor is the Hamiltonian density

$$
\mathcal{H}=\sum_{n} \pi^{(n)} \dot{\psi}^{(n)}-\mathcal{L}
$$

where

$$
\pi(t, \boldsymbol{x})=\frac{\partial \mathcal{L}\left(\psi(t, \boldsymbol{x}), \psi_{, \mu}(t, \boldsymbol{x})\right)}{\partial \dot{\psi}(t, \boldsymbol{x})}
$$

(see [9], pp. 15-16). The dependence of the Hamiltonian density $\mathcal{H}$ of (10) on the Lagrangian density $\mathcal{L}$ indicates that this Hamiltonian density is consistent with the Lagrangian density.

The energy-time uncertainty relation

$$
\Delta E \cdot \Delta t \geq \hbar
$$

restricts the accuracy of quantum expressions for energy. However, stable quantum states like that of a free particle or of an atomic ground state last a long time. Hence, a quantum theory should provide a consistent expression for energy, whose classical limit agrees with the classical value of energy. The Noether theorem proves that a Lagrangian density that does not depend explicitly on the space-time coordinates yields a Hamiltonian that conserves energy, momentum and angular momentum (see [9], pp. 17-19). If the analysis takes a covariant form then a consistent expression for the Hamiltonian also guarantees a consistent expression for momenta. Therefore, momentum issues are not discussed below.

The following experimental data illustrate the previous discussion. Consider the $e^{-} e^{+}$decay mode of the $Z$ boson in its rest frame [12]

$$
e^{-} \leftarrow Z \rightarrow e^{+}
$$

The outgoing $e^{-} e^{+}$leptons are measured by detectors and "so long as the particles involved in the reaction are outside the region of mutual interaction their mean motion can be described by classical mechanics" (see [2], p. 300). The $Z$ particle has a short half-life and its energy width is about $2.5 \mathrm{GeV}$ [12]. On the other hand, the outgoing $e^{-} e^{+}$leptons of (13) are stable particles that have a well-defined energy. Hence, also the $Z$ quantum theory must provide an appropriate expression for energy.

A classical equation of motion yields the particle's location. Hence, a quantum theory of an elementary massive particle should provide an expression for the particle's spatial density. In particular, electromagnetic interactions depend on local fields. Therefore, a quantum description of a charged particle depends on an appropriate expression for the spatial density of the charged quantum particle. 
The $Z$-decay process of (13) illustrates this issue. The experimental trajectories of the outgoing $e^{-} e^{+}$leptons are measured. These particles are identified as decay product of the $Z$ particle provided these trajectories intersect at a certain space-time point. The common spatial point of these trajectories should belong to the spatial region where the primary beams collide. It means that a theory of the $Z$ particle should provide an expression for density. An analogous conclusion holds for other elementary quantum particles.

A covariant expression for the quantum particle's density is derived from an application of the following version of the Noether theorem. Let $\alpha$ be a real variable. The invariance of the quantum field Lagrangian density under a global phase transformation $\exp (i \alpha)$ yields

$$
0=i \alpha\left[\frac{\partial \mathcal{L}}{\partial \psi}-\partial_{\mu}\left(\frac{\partial \mathcal{L}}{\partial\left(\partial_{\mu} \psi\right)}\right)\right] \psi+i \alpha \partial_{\mu}\left(\frac{\partial \mathcal{L}}{\partial\left(\partial_{\mu} \psi\right)} \psi\right)
$$

(see [13], p. 314). The overall value of the terms inside the square brackets vanishes due to the Euler-Lagrange equation. Furthermore, the variation parameter $\alpha \neq 0$ means that the expression inside the last brackets represent a conserved 4-current

$$
j_{, \mu}^{\mu}=0
$$

where

$$
j^{\mu}=\frac{\partial \mathcal{L}}{\partial\left(\partial_{\mu} \psi\right)} \psi
$$

Here $j^{0}$ is the required density.

The following list summarizes the constraints that are derived above.

C. 1 The equations of motion of a theory of an elementary quantum particle should be derived from an appropriate Lagrangian density.

C.2 A quantum theory should provide an expression for the Hamiltonian that is derived from this Lagrangian density.

C.3 The Lagrangian density of a quantum theory should have an interaction term.

C.4 A quantum theory should provide a consistent expression for density of the quantum particle.

\section{First Order Quantum Equations}

The Dirac equation of the electron is an example of a relativistic first order quantum equation. It is derived from this Lagrangian density

$$
\mathcal{L}_{D}=\bar{\psi}\left[\gamma^{\mu}\left(i \partial_{\mu}-e A_{\mu}\right)-m\right] \psi,
$$

where $A^{\mu}=(V, \boldsymbol{A})$ denote the electromagnetic 4-potential (see [9], p. 84, [11], p. 78). The term that contains $A_{\mu}$ is the interaction term of a charged Dirac particle.

The covariant form of the Dirac equation for $\psi$ is obtained from an 
application of the Euler-Lagrange Equation (7) to $\bar{\psi}$ of (17)

$$
\left[\gamma^{\mu}\left(i \partial_{\mu}-e A_{\mu}\right)-m\right] \psi=0
$$

(see [11], p. 42). Multiplying (18) by $\gamma^{0}$ and using the relations $\gamma^{0} \gamma^{0}=1$, $\gamma^{0} \gamma^{i}=\alpha_{i}$, one obtains the Hamiltonian form $H_{D}$ of the Dirac equation, where the Hamiltonian operates on the function $\psi$

$$
i \frac{\partial \psi}{\partial t}=H_{D} \psi=[\boldsymbol{\alpha} \cdot(-i \nabla-e \boldsymbol{A})+\beta m+e V] \psi
$$

(see [14], p. 11). This Hamiltonian is free of time-derivatives. Hence, the Dirac Equation (19) is an explicit first order partial differential equation.

The same form of the Hamiltonian is obtained from the general expression for the Hamiltonian density (10). The Hamiltonian density that is derived from (17) is

$$
\mathcal{H}=\pi \dot{\psi}-\mathcal{L}=\psi^{\dagger}[\boldsymbol{\alpha} \cdot(-i \nabla-e \boldsymbol{A})+\beta m+e V] \psi,
$$

where the relation $\bar{\psi}=\psi^{\dagger} \gamma^{0}$ is used. The Noether theorem (14) yields the 4-current of a Dirac particle

$$
j^{\mu}=\bar{\psi} \gamma^{\mu} \psi
$$

This 4-current satisfies the continuity equation

$$
j_{, \mu}^{\mu}=0
$$

(see [14], pp. 9, 23, 24). Hence, the Dirac density is $\psi^{\dagger} \psi$ and the Hamiltonian operator is factored out from the Dirac Hamiltonian density (20).

Solutions of the Dirac equation of a free electron and of the hydrogen atom are documented in textbooks (see e.g. [14], chapters 3, 4.4). These solutions fit experimental data. It can be concluded that the Dirac equation, namely the first order quantum equation, satisfies the requirements that are shown at the end of Section 2. Furthermore, this theory has a strong experimental support.

\section{Second Order Quantum Equations}

Consider the Lagrangian density of a second order quantum field equation

$$
\mathcal{L}=\phi_{, \mu}^{\dagger} \phi_{, v} g^{\mu v}-m^{2} \phi^{\dagger} \phi+O T
$$

where OT denotes other terms. This form is analogous to the Lagrangian density of the Klein-Gordon (KG) field (see e.g. [9], chapter 12), to that of the $W^{ \pm}, Z$ bosons (see e.g. [15], p. 309), and to that of the Higgs boson (see e.g. [11], p. 715). The first term of (23) is a product of derivatives of the quantum functions $\phi^{\dagger}, \phi$. Hence, the Euler-Lagrange Equation (7) yields second order derivatives of the $\phi^{\dagger}, \phi$ functions.

An observation of the Dirac Lagrangian density (17) and that of the second order quantum Equation (23) indicates an intrinsic difference between the quantum functions $\psi, \phi$, whose dynamical properties are derived from these Lagrangian densities. For example, the $\left[L^{-4}\right]$ dimension of a Lagrangian 
density proves that the dimension of a Dirac quantum function $\psi$ of (17) is $\left[L^{-3 / 2}\right]$. By contrast, the dimension of the quantum function $\phi$ of (23) is $\left[L^{-1}\right]$. This difference indicates that the impressive success of the Dirac theory cannot be automatically copied to second order theories of a quantum particle. Hence, a specific analysis of these theories is required.

First, let us examine mathematically real quantum functions. For example, see the real KG field ([9], p. 26), the $Z$ boson ([15], p. 309) and the Higgs boson (see [11] p. 715). The de Broglie hypothesis of the wave nature of a free massive particle is a fundamental principle of quantum theories (see [5], p. 3). This hypothesis says that the following relation holds between the particle's wave length and its linear momentum

$$
\lambda=2 \pi \hbar / p .
$$

The form of the factor that describes the undulating properties of the particle's wave function can be written as a linear combination of these expressions (see [5], p. 18)

$$
\sin (\boldsymbol{k} \cdot \boldsymbol{x}-\omega t), \cos (\boldsymbol{k} \cdot \boldsymbol{x}-\omega t), \exp \pm(\boldsymbol{k} \cdot \boldsymbol{x}-\omega t)
$$

Mathematically real functions can be written as a linear combination of the first and the second functions of (25). Hence, a real wave function of a free massive particle moving along the positive $x$-direction takes the form

$$
\psi(t, x)=A \sin (k x-\omega t-\delta),
$$

where $A$ is a real normalization factor and $\delta$ is a real constant. The free quantum particle that is analyzed here is massive and it has a rest frame. In this frame the particle's linear momentum is $p=k=0$, and its wave function (26) reduces to the form

$$
\psi(t, x)=A \sin (-\omega t-\delta) .
$$

It follows that for every integer $n$, the real wave function (27) vanishes identically throughout the entire 3 -dimensional space at the instant $t$ when $\omega t+\delta=n \pi$. This result means that the particle disappears at these instants. Therefore, no conserved expression for density can be defined for a mathematically real quantum function. This conclusion is consistent with the Noether expression for density (14). Indeed, (14) is based on the invariance of the Lagrangian density with respect to a multiplication by the complex factor $\exp (i \alpha)$. However, this complex factor is unacceptable for a mathematically real function. Furthermore, the fact that textbooks do not show a density expression for any mathematically real quantum function is another indication of the validity of this result. An analogous argument can be found in the following textbook (see [16], pp. 42, 43).

The Weinberg correspondence principle means that this result also holds for real QFT functions. The foregoing analysis proves that in the case of massive particles, mathematically real quantum functions are inconsistent with requirement 4 of Section 2. 
Let us examine mathematically complex second order quantum equations like those of the electrically charged KG particle and the $W^{ \pm}$bosons. The first term of (23) is a product of two space-time derivatives of the quantum function $\phi_{, \mu}^{\dagger}, \phi_{, \mu}$. The standard procedure of the introduction of an electromagnetic interaction is

$$
i \partial_{\mu} \rightarrow i \partial_{\mu}-e A_{\mu}
$$

(see [7], p. 343, [9], p. 84, [17], p. 198). Therefore, the electromagnetic part of the Lagrangian density (23) contains a product

$$
\mathcal{L}_{\mathrm{EM}}=e^{2} A^{\mu} A_{\mu}+O T .
$$

The first term of (29) is inconsistent with Maxwellian electrodynamics, because the Lagrangian density of this theory depends linearly on $A_{\mu}$ (see [3], p. $75,[18]$, p. 596). It means that the electroweak theory of the electrically charged $W^{ \pm}$particles lacks a consistent interaction term, and it violates the 3 constraint of Section 2. This outcome proves that an intrinsic error exists in second order quantum theories of an electrically charged particle.

\section{Discussion}

This section examines the present literature and presents further evidence of intrinsic inconsistencies of second order quantum equations that take the form of (23).

Let us examine the $W^{ \pm}$electroweak particles. The continuity equation

$$
j_{, \mu}^{\mu}=0
$$

is the mathematical expression of charge conservation. Here $j^{0}$ denotes charge density. Charge conservation is a crucial element of Maxwellian electrodynamics, which is recognized since the original work of Maxwell (see e.g. [18], pp. 217, 218). The electric 4-current is an element of the standard form of electromagnetic interaction (see [9], p. 84, [11] p. 78).

The following striking evidence demonstrates the different status of the Dirac equation and that of the second order electroweak equation of the electrically charged $W^{ \pm}$particles. A consistent expression for a conserved 4-current of the Dirac equation of the electron (and of its associated density) was found about one month after the publication of this equation [19] [20]. By contrast, many decades have elapsed since the birth of the electroweak theory, but textbooks still do not show a consistent expression for the $W^{ \pm}$4-current. Thus, for example, important research institutes like FermiLab and CERN use an effective expression for the $W^{ \pm}$electromagnetic interaction [21] [22].

Let us turn to another issue. The equation of motion of a quantum particle and its solution $\psi$ are fundamental elements of the theory's structure. For example, the compatibility between the theory and the relevant experimental data is based on the solution $\psi$ of the quantum equation. Furthermore, the Noether theorem guaranties that the theory satisfies conservation laws. An 
important element of the proof of this theorem is that $\psi$ satisfies the Euler-Lagrange equation of motion (see [7], p. 307, [9], p. 17).

Considering this issue, one finds a dramatic difference between the status of the Linear Dirac equation of Section 3 and that of the second order equations of Section 4. Indeed, an explicit form of the Dirac equation is shown in textbooks (see e.g. [7], p. 9, [14], pp. 6-9), and solutions of this equation fit the data (see, e.g. [14], pp. 52-60). By contrast, textbooks that discuss second order quantum theories of the $Z, W^{ \pm}$and of the Higgs boson do not show an explicit form of the Euler-Lagrange equation of the corresponding quantum theory. A fortiori, no physically acceptable solution of each of these equations is analyzed in textbooks.

The Higgs boson is coupled to other massive particles, and heavier particles dominate the process (see [11], p. 716). Therefore, the above mentioned $W^{ \pm}, Z$ discrepancies also affect the status of the Higgs boson.

Let us examine just one more issue. As pointed out above, the dimension of the quantum function $\phi$ of a second order equation is $\left[L^{-1}\right]$, whereas the dimension of density is $\left[L^{-3}\right]$. Hence, density of a second order quantum function must depend on a derivative. Relativistic covariance means that it depends on time-derivatives. Therefore, in the case of second order quantum functions, no consistent expression can be found for the Heisenberg picture, because this picture is based on functions that are time-independent. It means that any analysis that relies on the Heisenberg picture (see e.g. [7]) does not apply to second order quantum functions.

\section{Conclusions}

This work discusses the significance of the correspondence between classical physics, quantum mechanics and QFT. The crucial role of the Lagrangian density of an elementary quantum particle and of its Hamiltonian are pointed out. Well-known constraints on an acceptable quantum theory are explained in Section 2. The analysis proves that the first-order Dirac equation is compatible with these constraints. In contrast, the second-order quantum equations of the $\mathrm{KG}, W^{ \pm}, Z$ and the Higgs bosons are incompatible with these constraints.

It is interesting to mention that these results agree with Dirac's lifelong objection to second-order quantum equations (see [7], p. 14, [23], p. 3).

\section{Conflicts of Interest}

The author declares no conflicts of interest regarding the publication of this paper.

\section{References}

[1] Landau, L.D. and Lifshitz, E.M. (1960) Mechanics. Pergamon, Oxford.

[2] Goldstein, H., Poole, C. and Safko, J. (2002) Classical Mechanics. 3rd Edition, Addison Wesley, San Francisco, CA. 
[3] Landau, L.D. and Lifshitz, E.M. (2005) The Classical Theory of Fields. Elsevier, Amsterdam.

[4] Dirac, P.A.M. (1958) The Principles of Quantum Mechanics. Oxford University Press, London.

[5] Schiff, L.I. (1955) Quantum Mechanics. McGraw-Hill, New York.

[6] https://en.wikipedia.org/wiki/Correspondence principle

[7] Weinberg, S. (1995) The Quantum Theory of Fields, Vol. I. Cambridge University Press, Cambridge.

[8] Rohrlich, F. (2007) Classical Charged Particle. 3rd Edition, World Scientific, Singapore. https://doi.org/10.1142/6220

[9] Bjorken, J.D. and Drell, S.D. (1965) Relativistic Quantum Fields. McGraw-Hill, New York. https://doi.org/10.1063/1.3047288

[10] Merzbacher, E. (1970) Quantum Mechanics. John Wiley, New York.

[11] Peskin, M.E. and Schroeder, D.V. (1995) An Introduction to Quantum Field Theory. Addison-Wesley, Reading, MA.

[12] Tanabashi, M., et al. (2018) Particle Data Group. Physical Review D, 98, Article ID: 030001.

[13] Halzen, F. and Martin, A.D. (1984) Quarks and Leptons, an Introductory Course in Modern Particle Physics. John Wiley, New York.

[14] Bjorken, J.D. and Drell, S.D. (1964) Relativistic Quantum Mechanics. McGraw-Hill, New York.

[15] Weinberg, S. (1995) The Quantum Theory of Fields, Vol. II. Cambridge University Press, Cambridge.

[16] Berestetskii, V.B., Lifshitz, E.M. and Pitaevskii, L.P. (1982) Quantum Electrodynamics. Pergamon, Oxford.

[17] Pauli, W. and Weisskopf, V. (1934) The Quantization of the Scalar Relativistic Wave Equation. Helvetica Physica Acta, 7, 709-731. English Translation: Miller, A.I. (1994) Early Quantum Electrodynamics, University Press, Cambridge, 188-205.

[18] Jackson, J. D. (1975). Classical Electrodynamics. John Wiley, New York.

[19] Dirac, P.A.M. (1928) The Quantum Theory of the Electron. Proceedings of the Royal Society of London A, 117, 610.

[20] Darwin, C.G. (1928) The Wave Equations of the Electron. Proceedings of the Royal Society of London A, 118, 654.

[21] Abazov, V.M., et al. (2012) D0 Collaboration. Physics Letters B, 718, 451-459.

[22] Aad, G., et al. (2012) ATLAS Collaboration. Physics Letters B, 712, 289-462.

[23] Dirac, P.A.M. (1978) The Mathematical Foundations of Quantum Theory. In: Marlow, A.R., Ed., Mathematical Foundations of Quantum Theory, Academic Press, New York. https://doi.org/10.1016/B978-0-12-473250-6.50005-4 